BMJ Open

Respiratory

Research

\section{Comparison of oxygen uptake during cycle ergometry with and without functional electrical stimulation in patients with COPD: protocol for a randomised, single-blind, placebo- controlled, cross-over trial}

To cite: Medrinal C, Prieur G, Debeaumont $\mathrm{D}$, et al. Comparison of oxygen uptake during cycle ergometry with and without functional electrical stimulation in patients with COPD: protocol for a randomised, singleblind, placebo-controlled, cross-over trial. BMJ Open Resp Res 2016;3:e000130. doi:10.1136/bmiresp-2016000130

Received 2 February 2016 Revised 14 March 2016 Accepted 15 March 2016

CrossMark

For numbered affiliations see end of article.

\section{Correspondence to} Dr Clément Medrinal; medrinal.clement.mk@gmail. com

\section{ABSTRACT}

Introduction: Chronic obstructive pulmonary disease (COPD) has systemic repercussions that can lead to peripheral muscle dysfunction. Muscle atrophy reduces aerobic capacity, greatly limiting activities of daily living and quality of life. Pulmonary rehabilitation is the gold standard treatment for these patients, however, patients may not be able to reach sufficient training intensities for benefits to occur. Technologies such as functional electrical stimulation (FES) are currently being adapted and tested to enhance exercise training. We hypothesise that FES coupled with cycling (FEScycling) will improve maximal uptake of oxygen $\left(\mathrm{VO}_{2}\right)$ and aerobic capacity more than endurance training with placebo stimulation.

Methods: A randomised, single-blind, placebocontrolled crossover trial will be carried out to evaluate the effects of FES-cycling on $\mathrm{VO}_{2}$ during endurance exercise on a cycle ergometer in patients with COPD. 25 patients with COPD will carry out two 30 min sessions at a constant load; one session with active and one with placebo FES. The primary outcome is oxygen uptake recorded with a metabolic measurement system. Secondary outcomes include ventilation equivalent for oxygen, ventilation equivalent for carbon dioxide, cardiac output, lactate values, perceived dyspnoea and perceived muscle fatigue.

Results and conclusions: Approval has been granted by our Institutional Review Board (Comité de Protection des Personnes Nord-Ouest 3). The results of the trial will be presented at national and international meetings and published in peer-reviewed journals.

Trial registration number: NCT02594722.

\section{KEY MESSAGES}

Can a new concept of neuromuscular electrical stimulation (FES-cycling) improve metabolic and cardiovascular responses during endurance exercise in patients with chronic obstructive pulmonary disease (COPD)?

- To perform a preliminary study to evaluate cardiovascular and metabolic adaptations during FES-cycling endurance training in patients with COPD prior to evaluating the effects of a longterm programme.

> This study is the first to evaluate FES-cycling in COPD, which could become a new tool for pulmonary rehabilitation.

prevalence of $4-10 \%$ in the worldwide adult population. ${ }^{2}$ COPD is associated with systemic complications and peripheral muscle dysfunction, and is known to affect vital prognosis. ${ }^{3}$ Moreover, muscle fatigue occurs quickly and patients lack exercise tolerance, thus their quality of life is reduced. ${ }^{4} 5$ Multidisciplinary programmes combining cardiovascular training and patient education have been developed to reduce sedentariness and deconditioning. ${ }^{6}$

The effects of cardiovascular training depend on its intensity. ${ }^{7-9}$ Unfortunately, because of exertional dyspnoea and a limited exercise capacity, patients with COPD, and particularly those with severe COPD, may not be able to reach a high enough intensity for training to be beneficial. Maltais et $a l^{10}$ reported that the majority of patients included in their rehabilitation programme

\section{INTRODUCTION}

Chronic obstructive pulmonary disease (COPD) is a disabling condition with a were unable to maintain a high intensity of training. Current training strategies thus 
focus on increasing muscle work while avoiding increasing dyspnoea. ${ }^{7}$

Neuromuscular electrical stimulation may be a useful compromise for patients with high levels of dyspnoea, ${ }^{11-14}$ however, despite some advantages, the contractions produced are not very functional. ${ }^{15}{ }^{16}$ Functional electrical stimulation coupled with peddling, termed 'functional electrical stimulation cycling' (FES-cycling), was developed at the end of the 1980s. Since then, it has been used increasingly in patients with neurological disorders. ${ }^{17} 18$ The principle of FES-cycling is to electrically stimulate one or several muscle groups during an active or passive peddling task synchronised by a computer. ${ }^{19}$ It has been proposed as an alternative or complement to voluntary exercise for patients with neurological disorders, and has been shown to optimise training, and improve muscle strength and cardiovascular capacity. ${ }^{20}{ }^{21}$ Mutton et $a t^{22}$ showed a $10 \%$ increase in aerobic capacity in patients with spinal cord injury following a programme of FES-cycling. Increases in muscle volume have also been found in patients with spinal cord injury, using this technique. ${ }^{23}$

A recent study evaluated the effect of FES-cycling on the metabolic and cardiovascular responses of healthy patients. ${ }^{24}$ Oxygen uptake $\left(\mathrm{VO}_{2}\right)$ increased during the FES-cycling, as did cardiac frequency and lactate. These physiological results suggest that FES-cycling can improve cardiovascular and metabolic responses to exertion.

To the best of our knowledge, no studies have evaluated the potential benefits of FES-cycling in patients with COPD. Prior to evaluating the effects of a long-term programme, we propose to carry out a randomised, single-blind, placebo-controlled, cross-over trial to evaluate cardiovascular and metabolic adaptations during FES-cycling endurance training in patients with COPD.

\section{OBJECTIVE}

\section{Primary objective}

To evaluate the effect of FES-cycling on exertional $\mathrm{VO}_{2}$ compared with usual endurance training coupled with placebo-FES.

\section{Secondary objectives}

To evaluate the effect of FES-cycling on the respiratory equivalent for oxygen (ventilation $/ \mathrm{VO}_{2}$ ), the respiratory equivalent for carbon dioxide (ventilation $/ \mathrm{VCO}_{2}$ ), the ventilation $/ \mathrm{VCO}_{2}$ slope, cardiac output, lactate, perceived dyspnoea and perceived muscle fatigue.

\section{METHOD}

\section{Study design}

A single centre, randomised, single-blind, placebocontrolled, cross-over trial comparing $\mathrm{VO}_{2}$ during cycle-ergometer endurance exercise with active and placebo FES in patients with COPD. The study will be carried out in the functional exploration department of the Havre Hospital Group. The patients will carry out

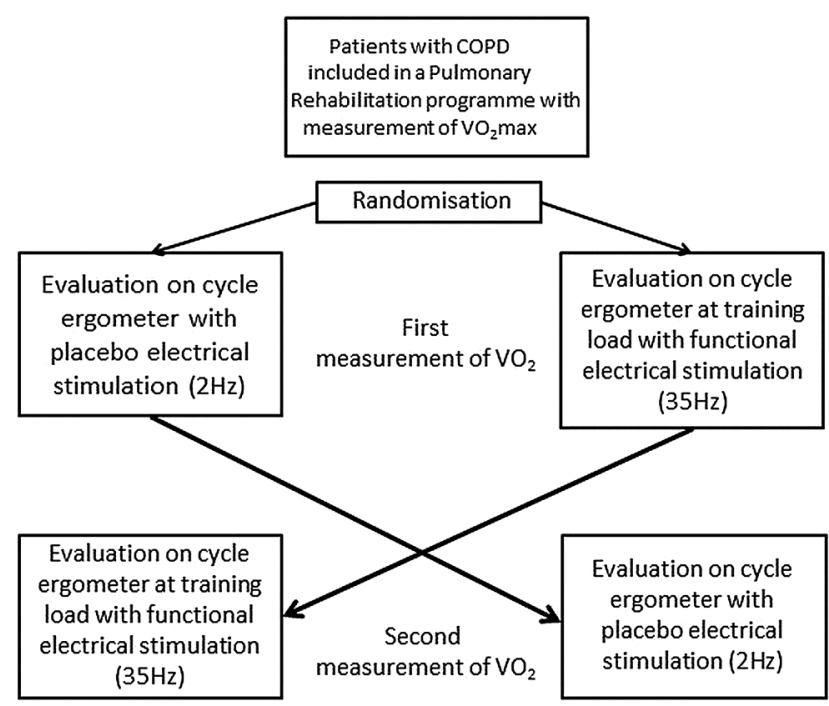

Figure 1 Study design. COPD, chronic obstructive pulmonary disease; $\mathrm{VO}_{2}$, maximal oxygen uptake.

two consecutive 30 min constant-load endurance sessions on a cycle ergometer with FES of the quadriceps muscle (FES-cycling session) and with placebo electrical stimulation (Control session). The order of the sessions will be randomised (figure 1).

\section{Participants \\ Inclusion criteria}

Patients with GOLD stage 2, 3 or 4 COPD participating in a respiratory rehabilitation programme. Patients with no changes to $\beta$-blocker treatment in the past 3 months. Patients who agree to participate voluntarily and who sign the informed consent form.

\section{Exclusion criteria}

- Patients with a pacemaker.

- Patients who have had an exacerbation within the past 4 weeks.

- Patients with other conditions that may affect their participation in rehabilitation (osteoarticular or neuromuscular disorders or severe psychiatric disorders, patients with severe anaemia $(<8 \mathrm{~g} / \mathrm{dL})$.

- Patients with central neurological pathology.

- Patients on non-invasive night-time mechanical ventilation.

- Patients who are unable to carry out $30 \mathrm{~min}$ of exercise.

\section{Recruitment}

Prior to participating in the respiratory rehabilitation programme at the Havre Hospital Group, patients will undergo an initial exercise test including measurements of $\mathrm{VO}_{2}$ max, the 6 min walk test (6MWT) and a cardiorespiratory assessment to screen for contraindications to exercise. $\mathrm{VO}_{2}$ max will be evaluated during a triangular effort test with increasing load, on an ergometer. Following a 2 min warm-up, the intensity will be 
increased by $10 \mathrm{~W}$ every minute. The test will be carried out under the surveillance of a pulmonologist and a cardiologist. As is customary in our training programmes, the load for the endurance session will be set as the load at which ventilatory threshold was reached during the exercise test.

Patients who fulfil the inclusion criteria will be informed of the protocol. Those who wish to participate will receive all the necessary information. The date of the evaluation will be determined at least 4 weeks after the signing of the informed consent form, and a maximum of 6 weeks after the initial exercise test.

\section{Randomisation}

The order of the tests will be randomised using independent methods. Randomisation will be carried out by the Clinical Research Unit via computer software. The investigator will receive the randomly generated treatment allocation in a sealed envelope just before the endurance session.

\section{Intervention}

$\mathrm{VO}_{2}$ will be measured during $30 \mathrm{~min}$ of endurance exercise with functional electrical stimulation using the Reha Stim device. Two RehaMove electrodes $(5 \times 9 \mathrm{~cm})$ will be positioned at each extremity of both quadriceps muscles to stimulate both muscles. A rectangular, intermittent, bidirectional current with no ramp will be used and the intensity will be modulated to obtain a palpable muscle contraction. The other electrical stimulation parameters will be identical for all patients (length: $300 \mu$ s, frequency: $35 \mathrm{~Hz}$. These settings are based on usual electrostimulation protocols ${ }^{15}$ ).

During the ergometric cycling, the stimulator will be controlled by a personal computer. The software will ensure that muscle contractions are induced at the appropriate pedal angles during knee extension.

Following a 2 min warm-up, the load will be increased to reach the training load determined during the initial exercise test. The patients will peddle at a frequency of 50-60 rotations per minute for 30 min. ${ }^{12}$

At the end of the session, a capillary measurement of lactate will be taken using 'lactatepro II'. The patient will be asked to evaluate his/her dyspnoea on the Borg scale and muscle fatigue on a visual analogue scale.

\section{Control}

$\mathrm{VO}_{2}$ will be measured during 30 min of endurance exercise with placebo electrotherapy that does not influence ventilation (length $300 \mu$ s, frequency $2 \mathrm{~Hz}$ ). ${ }^{25}$ The intensity will be low, so as not to produce any muscle contraction.

Conditions will be identical for both endurance sessions and patients will have a 30 min rest between each. The training load will be identical for both endurance sessions.

\section{Blinding}

The use of placebo electrotherapy means that patients can be blind to their group. The therapist who sets up the equipment will not be blind; however, both the pulmonologists carrying out the data analysis will be blind to the allocations.

\section{DATA COLLECTION}

\section{Oxygen uptake}

The primary outcome will be oxygen uptake $\left(\mathrm{VO}_{2}\right)$ throughout the test. $\mathrm{VO}_{2}$ will be continuously measured using a metabolic measurement system (Vmax Spectra 29) that carries out a breath-by-breath gas analysis. The mean is calculated every $30 \mathrm{~s}$. A pulmonologist (BL) and a physiotherapist (either CM, GP, ARQ or YC) will review all test results.

\section{Ventilation equivalent for oxygen}

The ventilation equivalent for oxygen (ventilation/ $\mathrm{VO}_{2}$ ratio) will be used to determine the number of litres of air required to obtain $1 \mathrm{~L}$ of oxygen.

\section{Ventilation equivalent for carbon dioxide}

The ventilation equivalent for carbon dioxide (ventilation $/ \mathrm{VCO}_{2}$ ratio) will be used to determine the number of litres of air required to eliminate $1 \mathrm{~L}$ of carbon dioxide.

\section{Cardiac output}

The linear relationship between the adjustment of cardiac output and the increase in oxygen consumption will be used to estimate cardiac output $(\mathrm{L} / \mathrm{min})$. The following equation will be used to estimate cardiac output during the endurance session: $5.5 \times \mathrm{VO}_{2}(\mathrm{~L} / \mathrm{min})+5 .{ }^{26} 27$

\section{Lactate}

Capillary blood lactate values will be measured at the end of each endurance session. The Lactate Pro II device will be used. With this device, the measurement takes only $15 \mathrm{~s}$ and requires only $0.3 \mu \mathrm{L}$ of blood. ${ }^{28}$

\section{Dyspnoea}

Perceived dyspnoea will be measured using the Borg scale, at the end of each endurance session.

\section{Muscle fatigue}

Perceived muscle fatigue will be measured using a visual analogue scale at the end of each endurance session.

\section{POWER CALCULATION AND SAMPLE SIZE}

$\mathrm{VO}_{2}$ will be measured using an infrared gas analyser. Based on the results of previous studies, ${ }^{29} 22$ pairs of patients should be included to detect a difference in mean $\mathrm{VO}_{2}$ between groups of $200 \mathrm{~mL}$ and to reject the null hypothesis with a power of $90 \% .^{30}$ The associated type I probability error is 0.05 . We plan to include $10 \%$ more participants, thus 25 patients in total. 


\section{STATISTICAL ANALYSIS}

Normally distributed data will be expressed as means $( \pm$ SDs $)$ and non-normally distributed data will be expressed as medians (and IQRs).

The values of $\mathrm{VO}_{2}, \mathrm{VCO}_{2}$ and $\mathrm{VE}$ will be collected breath-by-breath and means will be calculated every 5 min. Within-group data will be analysed using paired $\mathrm{t}$ tests or Wilcoxon signed rank tests. Analysis of variance will be used to compare changes during the sessions between groups. A Bonferroni t test will used post hoc. The level of significance will be set at $\leq 0.05$.

\section{STRENGTHS AND LIMITATIONS}

This study is the first to evaluate the effects of FES in patients with COPD.

The use of a placebo will reduce the risk of interpretation bias.

There are two main limitations in this study. First, it is single centre. Second, the evaluation of a single session does not provide information regarding long-term clinical effectiveness; however, the results will provide a basis for such a study.

\section{Author affiliations}

${ }^{1}$ Pulmonology Department, Groupe Hospitalier du Havre, Montivilliers, France ${ }^{2}$ Groupe de Recherche sur le Handicap Ventilatoire, UPRES EA 3830, HauteNormandie Institute of Biomedical Research and Innovation, Rouen University, Rouen, France

${ }^{3}$ Unité de Physiologie Respiratoire et Sportive, Hôpital de Bois Guillaume, $\mathrm{CHU}$ de Rouen, Rouen, France

${ }^{4}$ Intensive Care Unit Department, Groupe Hospitalier du Havre, Montivilliers, France

${ }^{5}$ Physiotherapy Department, Groupe Hospitalier du Havre, Montivilliers, France ${ }^{6}$ Pulmonology Department, Hôpital Jacques Monod, Montivilliers, France ${ }^{7}$ University of Applied Sciences and Arts Western Switzerland (HES-SO), Lausanne, Switzerland

${ }^{8}$ Intensive Care Unit, Respiratory Department, Rouen University Hospital, Rouen, France

${ }^{9}$ Groupe de Recherche sur le Handicap Ventilatoire, UPRES EA 3830, HauteNormandie Institute of Biomedical Research and Innovation, Rouen University, Rouen, France

Contributors CM, GP, OC and BL were responsible for trial concept and design. $\mathrm{CM}, \mathrm{GP}, \mathrm{ARQ}, \mathrm{YC}$ and $\mathrm{BL}$ were responsible for acquisition of data. $\mathrm{DB}$ and $\mathrm{JQ}$ analysis and interpretation of data and drafting the article. $\mathrm{CM}, \mathrm{DB}, \mathrm{OC}$ and $\mathrm{BL}$ revising it critically for important intellectual content and final approval of the version to be published. CM had full access to all trial data and takes responsibility for the integrity of the data and the accuracy of the data analysis.

Funding This work was supported by ADIR Association.

Competing interests None declared.

Ethics approval CPP Nord-Ouest 3.

Provenance and peer review Not commissioned; externally peer reviewed.

Ethics and dissemination The study has been approved by our Institutional Review Board (Comité de Protection des Personnes Nord-Ouest 3). In conformity with the Declaration of Helsinki, all participants will be recruited to participate voluntarily and they will sign a written informed consent form. The results of the trial will be presented at national and international meetings and published in peer-reviewed journals. Results will be registered with ClinicalTrials.gov. We will also disseminate the main results in a letter to all participants. The study has been registered with ClinicalTrials.gov (NCT02594722)
Open Access This is an Open Access article distributed in accordance with the Creative Commons Attribution Non Commercial (CC BY-NC 4.0) license, which permits others to distribute, remix, adapt, build upon this work noncommercially, and license their derivative works on different terms, provided the original work is properly cited and the use is non-commercial. See: http:// creativecommons.org/licenses/by-nc/4.0/

\section{REFERENCES}

1. Buist AS, McBurnie MA, Vollmer WM, et al. International variation in the prevalence of COPD (the BOLD Study): a population-based prevalence study. Lancet 2007;370:741-50.

2. Lozano R, Naghavi M, Foreman K, et al. Global and regional mortality from 235 causes of death for 20 age groups in 1990 and 2010: a systematic analysis for the Global Burden of Disease Study 2010. Lancet 2012;380:2095-128.

3. Marquis K, Debigaré R, Lacasse $\mathrm{Y}$, et al. Midthigh muscle cross-sectional area is a better predictor of mortality than body mass index in patients with chronic obstructive pulmonary disease. $\mathrm{Am} \mathrm{J}$ Respir Crit Care Med 2002;166:809-13.

4. Gosselink R, Troosters T, Decramer M. Peripheral muscle weakness contributes to exercise limitation in COPD. Am J Respir Crit Care Med 1996;153:976-80.

5. Decramer M, Gosselink R, Troosters T, et al. Muscle weakness is related to utilization of health care resources in COPD patients. Eur Respir J 1997;10:417-23.

6. Lacasse Y, Goldstein R, Lasserson TJ, et al. Pulmonary rehabilitation for chronic obstructive pulmonary disease. Cochrane Database Syst Rev 2006;(4):CD003793.

7. Casaburi R, Patessio A, loli $F$, et al. Reductions in exercise lactic acidosis and ventilation as a result of exercise training in patients with obstructive lung disease. Am Rev Respir Dis 1991:143:9-18.

8. Gimenez M, Servera E, Vergara $P$, et al. Endurance training in patients with chronic obstructive pulmonary disease: a comparison of high versus moderate intensity. Arch Phys Med Rehabil 2000;81:102-9.

9. Punzal PA, Ries AL, Kaplan RM, et al. Maximum intensity exercise training in patients with chronic obstructive pulmonary disease. Chest 1991;100:618-23.

10. Maltais F, LeBlanc P, Jobin J, et al. Intensity of training and physiologic adaptation in patients with chronic obstructive pulmonary disease. Am J Respir Crit Care Med 1997;155:555-61.

11. Sillen MJ, Franssen FM, Delbressine JM, et al. Efficacy of lower-limb muscle training modalities in severely dyspnoeic individuals with COPD and quadriceps muscle weakness: results from the DICES trial. Thorax 2014:69:525-31.

12. Spruit MA, Singh SJ, Garvey C, et al. An official American Thoracic Society/European Respiratory Society statement: key concepts and advances in pulmonary rehabilitation. Am J Respir Crit Care Med 2013;188:e13-64

13. Vivodtzev I, Pépin JL, Vottero G, et al. Improvement in quadriceps strength and dyspnea in daily tasks after 1 month of electrical stimulation in severely deconditioned and malnourished COPD. Chest 2006;129:1540-8.

14. Vivodtzev I, Debigaré R, Gagnon P, et al. Functional and muscular effects of neuromuscular electrical stimulation in patients with severe COPD: a randomized clinical trial. Chest 2012;141:716-25.

15. Pan L, Guo Y, Liu X, et al. Lack of efficacy of neuromuscular electrical stimulation of the lower limbs in chronic obstructive pulmonary disease patients: a meta-analysis. Respirology 2014;19:22-9.

16. Vivodtzev I, Lacasse Y, Maltais F. Neuromuscular electrical stimulation of the lower limbs in patients with chronic obstructive pulmonary disease. J Cardiopulm Rehabil Prev 2008;28:79-91.

17. Glaser RM, Figoni SF, Collins SR, et al. Physiologic responses of $\mathrm{SCl}$ subjects to electrically induced leg cycle ergometry. IEEE Conf Eng Med Biol Soc 1988;1638-40.

18. Hooker S, Figoni S, Glaser R, et al. Physiologic responses to prolonged electrically stimulated leg-cycle exercise in the spinal cord injured. Arch Phys Med Rehabil 1990;7:863-9.

19. Fornusek $C$, Davis $G$, Sinclair $P$, et al. Development of an isokinetic functional electrical stimulation cycle ergometer. Neuromoduation 2004;1:56-64.

20. Davis G, Hamzaid N, Fornusek C. Cardiorespiratory, metabolic, and biomechanical responses during functional electrical stimulation leg exercise: health and fitness benefits. Artif Organs 2008;32:625-9.

21. Janssen T, Glaser R, Shuster D. Clinical efficacy of electrical stimulation exercise training: effects on health, fitness, and function. Top Spinal Cord Inj Rehab 1998;3:33-49. 
22. Mutton DL, Scremin AM, Barstow TJ, et al. Physiologic responses during functional electrical stimulation leg cycling and hybrid exercise in spinal cord injured subjects. Arch Phys Med Rehabil 1997;78:712-18.

23. Griffin L, Decker MJ, Hwang JY, et al. Functional electrical stimulation cycling improves body composition, metabolic and neural factors in persons with spinal cord injury. J Electromyogr Kinesiol 2009:19:614-22.

24. Watanabe K, Taniguchi Y, Moritani T. Metabolic and cardiovascular responses during voluntary pedaling exercise with electrical muscle stimulation. Eur J Appl Physiol 2014;114:1801-7.

25. Minogue CM, Caulfield BM, Lowery MM. Whole body oxygen uptake and evoked knee torque in response to low frequency electrical stimulation of the quadriceps muscles: VO2 frequency response to NMES. J Neuroeng Rehabil 2013;10:63.
26. Jones NL. Clinical exercise testing. 4th edn. Philadelphia: WB Saunders, 1997.

27. Perrault $\mathrm{H}$, Richard R. Clinical exercise testing and the Fick equation: strategic thinking for optimizing diagnostis. Rev Mal Respir 2012;29:501-20.

28. Bonaventura JM, Sharpe K, Knight E, et al. Reliability and accuracy of six hand-held blood lactate analysers. J Sports Sci Med 2015;14:203-14.

29. Saey D, Lemire BB, Gagnon P, et al. Quadriceps metabolism during constant workrate cycling exercise in chronic obstructive pulmonary disease. J App Physiol (1985) 2011;110:116-24 .

30. Medrinal C, Prieur G, Hemeryck B, et al. Metabolic effects of electrotherapy combined with bedside cycle-ergometry: preliminary study. Kinesither Rev 2015;15:37-41. 